\title{
Rupture process of the 2007 Notohanto earthquake by using an isochrones back-projection method and K-NET/KiK-net data
}

\author{
Nelson Pulido, Shin Aoi, and Hiroyuki Fujiwara \\ National Research Institute for Earth Science and Disaster Prevention, Japan \\ (Received June 30, 2007; Revised February 27, 2008; Accepted March 24, 2008; Online published November 7, 2008)
}

\begin{abstract}
We estimated the source process of the 2007/3/25 Notohanto earthquake using a new method for source imaging based on an "isochrones-backprojection" of observed seismograms in the source region (IBM). The IBM differs from conventional earthquake source modeling approaches in that no inversion procedures are required. The idea of IBM is to directly back-project amplitudes of seismogram envelopes around the source into a space image of the earthquake rupture. The method requires the calculation of isochrones times at every station used for source imaging, for a set of grids points distributed within the source fault plane. Total grid "brightness" is calculated by adding all observed waveform envelope amplitudes at every station, for every isochrone line crossing the grid, in order to produce an image of the total fault plane brightness distribution. Our source imaging results of the Notohanto earthquake show two large brightness regions; the first region is located $10 \mathrm{~km}$ above the hypocenter, and the second region is located at the bottom of the northern end of the fault plane. These regions approximately correspond to large slip areas obtained by a conventional inversion approach. Our method has the capability to quickly map asperities of large earthquakes using observed strong motion data.
\end{abstract}

Key words: Source process, array imaging, high frequency, Notohanto earthquake.

\section{Introduction}

The 2007 Notohanto earthquake $\left(M_{\mathrm{w}}=6.7,2007 / 3 / 25\right.$, JT 09:41:57.9) was located by the Japan Meteorological Agency (JMA) at a latitude of 37.220, longitude of 136.685 and depth of $11 \mathrm{~km}$. According to the National Research Institute for Earth Science and Disaster Prevention broadband network (F-NET, NIED), the earthquake had a reverse mechanism with a highly oblique slip direction (strike $58^{\circ}$, $\operatorname{dip} 66^{\circ}$ and rake $132^{\circ}$ ). The Notohanto earthquake recorded JMA instrumental intensities up to 6 (upper), and ground accelerations and velocities reached values of 850 gals and 100 kines respectively, at NIED, K-NET sites. The earthquake ground shaking induced a massive damage to houses and roads and triggered many rock and embankment failures around the source (Hamada et al., 2007).

Standard methodologies for calculation of the earthquakes source process are based on inversion procedures which require the calculation of complete source-stations Greens functions (i.e. Hartzell and Heaton, 1983; Sekiguchi et al., 2002; and others). Alternative procedures have been developed in order to directly retrieve an image of the rupture process from high-frequency seismograms around the source (Spudich and Cranswick, 1984; Ellsworth, 1992; Ishii et al., 2005; Fletcher et al., 2006; Kao and Shan, 2007; Allmann and Shearer, 2007). Other procedures have implemented the concept of isochrones introduced by Bernard and Madariaga (1984) and Spudich and Frazer (1984) to obtain a map of the fault slip from the back-projection of near-

Copyright (c) The Society of Geomagnetism and Earth, Planetary and Space Sciences (SGEPSS); The Seismological Society of Japan; The Volcanological Society of Japan; The Geodetic Society of Japan; The Japanese Society for Planetary Sciences; TERRAPUB source seismograms (Iwata and Irikura, 1989; Festa, 2004; Festa and Zollo, 2006). Festa and Zollo (2006) applied this method to investigate the source process of the 2000 Tottori earthquake from near-source low frequency seismograms.

In this study we extend the methodology of Festa and Zollo (2006) by incorporating the use of high-frequency (HF) seismograms for imaging the source rupture. To facilitate the use of HF waveforms we calculate envelopes of velocity seismograms to image the fault plane brightness by constraining the fault rupture to a constant value. We apply the isochrones back-projection method (IBM) to obtain an image of the large brightness regions across the fault plane of the 2007 Notohanto earthquake. We found that our methodology is capable of quickly map asperities of large to moderate earthquakes by using strong motion waveforms and is able to provide stable estimates of the fault rupture velocity.

\section{The Isochrones Back-projection Method}

The main idea of the IBM is to directly back-project amplitudes of seismograms envelopes around the source into a space image of the earthquake rupture. The method requires the calculation of theoretical travel times between a set of grid points distributed across the fault plane and every station, which are adjusted by a station correction factor, for a 1D velocity model. Next we calculate the rupture time of every grid within the fault plane by assuming some arbitrary constant rupture velocity value, and obtain the isochrones times for every station. We select waveforms that have clear $P$ - and $S$-wavelets, which means stations located approximately between $40 \mathrm{~km}$ and $100 \mathrm{~km}$ from the epicenter. We extract $P$-wave windows between the 

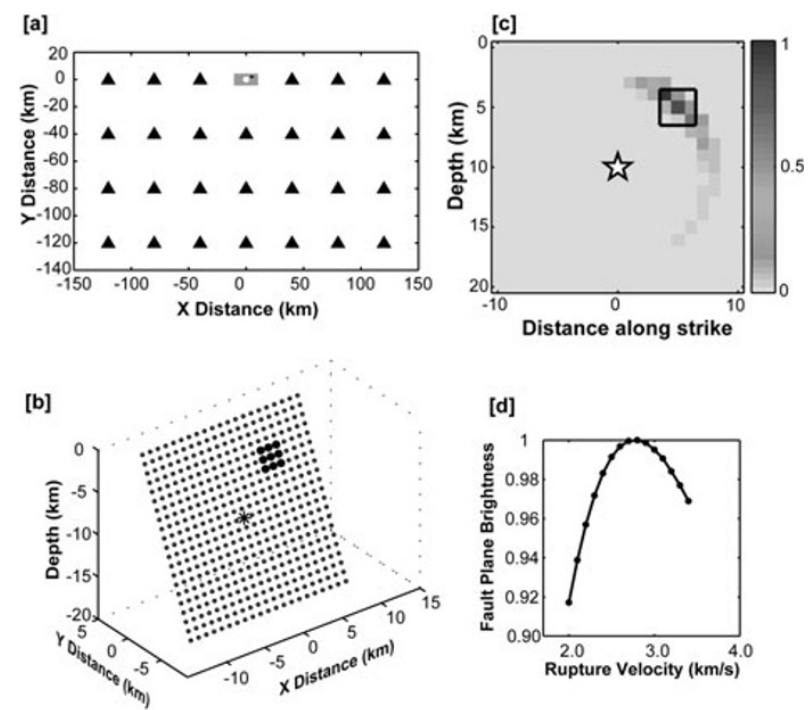

Fig. 1. (a) Station distribution used for the resolution test. The stations are uniformly spaced at $40 \mathrm{~km}$, and the source is located at the origin. (b) Fault plane used for the source imaging. The plane is dipping $66^{\circ}$ downwards the $Y$-axis. The black dots represent the asperity location used to calculate the synthetic seismograms. (c) Results of the IBM source imaging (normalized fault brightness) for a rupture velocity value of 2.5 $\mathrm{km} / \mathrm{s}$ (color scale). The assumed asperity is enclosed by a black square. (d) Rupture velocity vs normalized total fault plane brightness.

origin time of the earthquake and the theoretical arrival of the $S$-wave, taper $1 \mathrm{~s}$ of the waveforms towards the end, and apply a band-pass filter between 1 and $30 \mathrm{~Hz}$. Velocity envelopes $(V)$ are calculated using the root-mean-square of the original seismograms and their Hilbert transform $(H)$,

$$
V[v(t)]=\left(v(t)^{2}+H^{2}[v(t)]\right)^{2}
$$

where $v(t)$ are the velocity waveforms. The total grid brightness $\left(E_{g}\right)$ is calculated by adding averaged envelope amplitudes, for a window centered at the grid isochrone time for all stations,

$$
E_{g}=\sum_{i=1}^{N}\left[\sum_{k=-W}^{W} A\left(t_{g i}+t_{g}^{\mathrm{rup}}+k \Delta t+c_{i}\right)\right] \frac{R_{g i} w_{i}}{2 W}
$$

where $A$ is the envelope value, $N$ is the total number of stations, $W$ is the envelope averaging window half width, $t_{g i}$ is the travel time between grid $g$ and station $i, t_{g}^{\text {rup }}$ is the grid rupture time, $\Delta t$ is the sampling time, $c_{i}$ is the station correction time for the $i$ station, and $R_{g i}$ is the source-station ray length, intended as a correction for geometrical spreading. We apply appropriate data weighting factors $w_{i}$, which can be representative of the quality of the data. In this case, we assign the weights a value proportional to the station epicentral distance, in order to taper migration artifacts produced by stations towards the perimeter of the array (Allmann and Shearer, 2007). The calculation of grid brightness in Eq. (2), is similar to the envelope averaging procedure of Kao and Shan (2007), but introduces the concept of isochrones times to constraint the fault rupture. We calculate the total brightness for all grids across the fault plane and normalize the values by the maximum grid brightness. In this way we obtain the brightness distribution across the fault plane, which could be indicative of the asperity location as we will describe in a later section.

In our imaging procedure the grid brightness is directly related to the final slip, as our method is based on the isochrones theory, in which the ground motion amplitude at a given time and location can be approximately calculated as the line integral of slip along the corresponding isochrone (Spudich and Frazer, 1984). The imaged grid brightness does not exactly correspond to the final slip, as we are using envelopes instead of directly using seismograms for the source imaging, and the isochrones method is based on the assumption of an instantaneous slip. However, a comparison between the IBM source imaging results with the final slip obtained from conventional inversion procedures may be useful to determine a scaling relationship between grid brightness and fault slip. This may be a subject for future research.

\section{Source Image Post-processing}

The source images obtained by the IBM are usually characterized by a defocusing along the isochrones lines, mainly arising from the assumption of a uniform brightness distribution along the isochrones as well as poor azimuth station coverage, as pointed out by Festa and Zollo (2006). To remove this effect, we apply the "restarting" procedure described in Festa and Zollo (2006) to the backprojected images of brightness. This procedure consists of using the result of the first back-projected images as a priori information for a new back-projection. Festa and Zollo (2006) found that the application of the restarting iterative procedure to back-projected images of the 2000 Tottori, Japan earthquake lead to a significant improvement of the observed-synthetic data fit. The number of iterations will depend on an observation-synthetic fit criterion. In our methodology we do not calculate forward envelopes in order to allow for a quick mapping of the source brightness, and therefore, we adopt an iteration value that allows us to sufficiently reduce the defocusing effect. On the other hand, the introduction of a specific source-station ray length in Eq. (2), intended as a simple correction for geometrical spreading, also contributes to reducing the defocusing effect along the isochrones.

\section{Resolution Test}

To explore the resolution of the resolved source images by the IBM, we prepared a synthetic test, as shown in Fig. 1. We assumed a uniform distribution of 27 stations, spaced at $40 \mathrm{~km}$, and covering two quadrants around the source area, to reproduce a similar station coverage as for the Notohanto earthquake (Fig. 1(a)). Synthetic seismograms at every station are calculated from a fault plane with a small asperity, as shown in Fig. 1(b), dipping downwards the $Y$ axis with the same dip angle as the value for the Notohanto earthquake $\left(66^{\circ}\right)$. Point sources are uniformly distributed every kilometer across the fault plane, and we assume that only the grids corresponding to the asperity radiate waves. We specify a uniform fault rupture starting at a depth of $11 \mathrm{~km}$ and at the $X-Y$-axis origin, with a value of $2.5 \mathrm{~km} / \mathrm{s}$. We assume for simplicity that the ground motion generated from each grid at every station consists of positive and neg- 


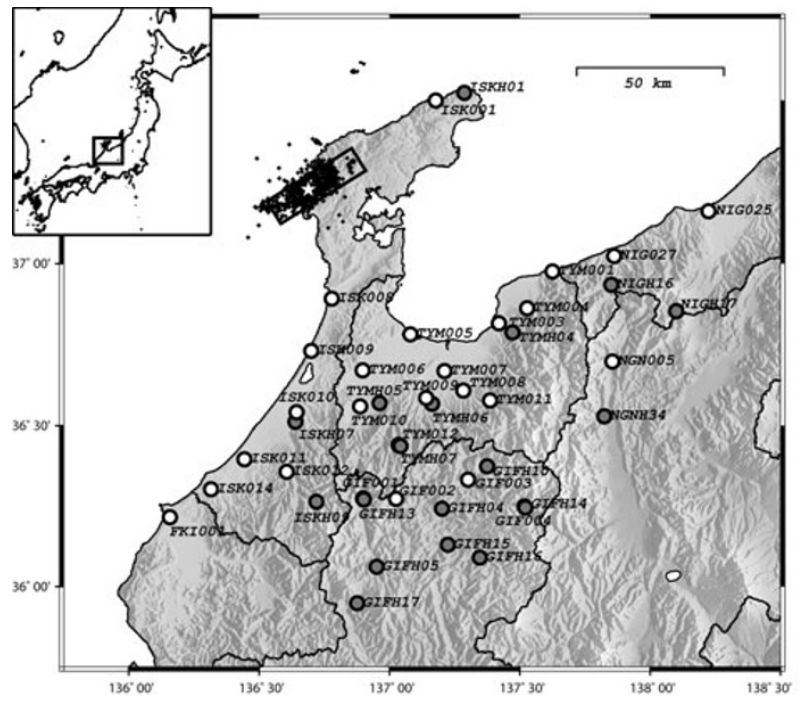

Fig. 2. K-NET and KiK-net stations used for the source imaging of the 2007 Notohanto earthquake $\left(M_{\mathrm{w}} 6.7\right)$, depicted as white and gray circles, respectively. The crosses represent the first $24 \mathrm{~h}$ of aftershocks with magnitude larger than 2. A white star depicts the JMA epicenter of the mainshock, and a black rectangle the fault plane model used for source imaging.

ative adjacent triangles with a total duration of $0.4 \mathrm{~s}$, which would approximately correspond to the far-field velocity radiation of a ramp dislocation at the source. We normalize the pulse by its maximum amplitude and divide it by the epicentral distance as an approximation for geometrical spreading. The arrival time of the pulse at every station is specified by the isochrone time, which is calculated for a uniform rupture velocity and for a half-space with a $P$ wave velocity of $6 \mathrm{~km} / \mathrm{s}$. Total ground motion at every station is calculated by adding the contribution from all point sources. To simulate realistic conditions we incorporate a random site amplification to each station and add a $20 \%$ of normal noise.

We apply the methodology for source imaging described in Sections 2 and 3, using the synthetic waveforms and the grid geometry in Fig. 1(b). The results of source imaging are shown in Fig. 1(c). We can observe that the IBM is able to illuminate the assumed asperity location, depicted as a black rectangle in Fig. 1(c). We also performed the IBM source imaging for a random distribution of stations covering a similar area than the configuration in Fig. 1(a), and found that the asperity location was imaged unequivocally. Although the asperity location is correctly identified in all cases, we have found that the imaged source has an elongated Kernel along the imaged asperity. This feature may result from poor station coverage as well as the assumption of an instantaneous slip in the isochrones method (Spudich and Frazer, 1984). On the other hand, we have found that using a distribution of stations located closer that $40 \mathrm{~km}$ around the source area, for various spacing values, always lead to a wrong imaging of the asperity location. This result can be useful for the station selection process for the source imaging of actual earthquakes.

We repeated the source imaging for the fault and station configuration in Fig. 1, but assuming different values of

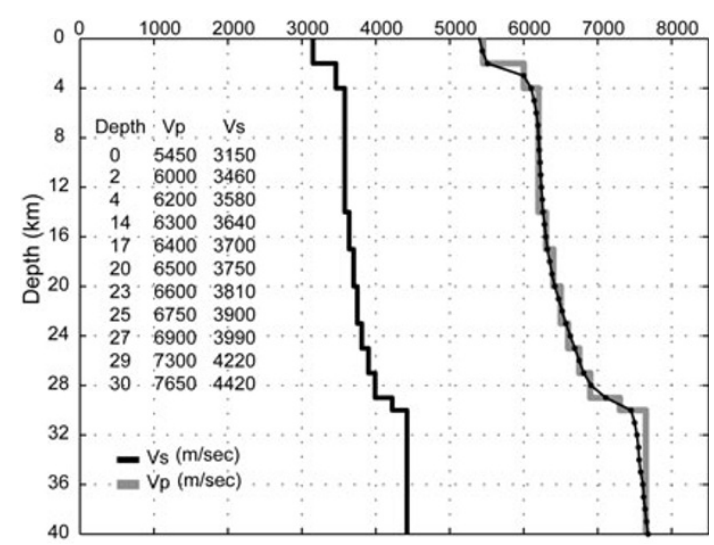

Fig. 3. Velocity model for the Atotsugawa fault region (Ito and Wada, 2002), depicted as a continous black line with dots. The multilayered model show as a gray line is used for the calculation of travel times for the source imaging of the Notohanto earthquake.

fault rupture velocity. We found that the total fault plane brightness, obtained by adding the brightness of all grids across the fault plane, have a maximum for a value close to the rupture velocity used for the synthetic waveforms calculation (Fig. 1(d)). This result may be explained by the fact that when no radiation is occurring at the imaged grid, the summation of envelopes at all stations will not be coherent. In other words, the overall summation of envelopes will reach a maximum for a rupture velocity that approaches the actual value.

\section{Notohanto Earthquake Data}

We used waveforms of the Notohanto earthquake recorded at 44 NIED strong motion stations (26 K-NET, and $18 \mathrm{KiK}$-net) for the source imaging of the earthquake (Fig. 2). We selected records with distinct $P$ - and $S$ wavelets, which corresponded in general to stations with epicentral distances ranging from 40 to $150 \mathrm{~km}$. In order to calculate travel times, we set a mesh of point sources uniformly spaced at $2 \mathrm{~km}$, across a plane with dip and strike angles corresponding to the F-NET solution (strike N58 ${ }^{\circ}$, dip $66^{\circ}$ ), intersecting the JMA hypocenter solution. The shallower row of point sources is located at a depth of $1.89 \mathrm{~km}$, and the grid-mesh covers a rectangular area of $22 \mathrm{~km}$ along dip and $34 \mathrm{~km}$ along strike, as shown in Fig. 2. We calculated all travel times between grids and stations using a velocity model developed for the Atotsugawa fault region (Ito and Wada, 2002) (Figs. 3 and 4). For simplicity, the original velocity was parameterized as a multilayered model with no gradients (Fig. 3). All grid travel times were adjusted by a station correction factor, obtained by calculating the difference between observed and calculated travel times at all stations for an M 5.3 aftershock (2007/03/25, 18:11:00).

\section{Results}

\subsection{Stations corrections}

In Fig. 4 we show the station corrections for the $P$-waves, obtained by using an $M 5.3$ aftershock. We can observe that the correction oscillates in a range from $-0.35 \mathrm{~s}$ to $1 \mathrm{~s}$. The stations with the largest corrections are located in the Toyama and Kaga plains where large site effects 


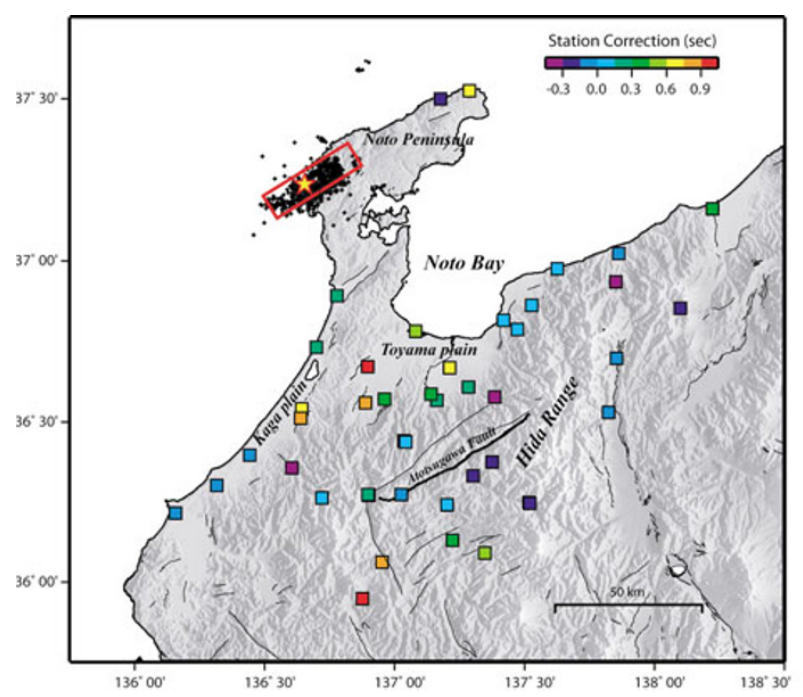

Fig. 4. Station corrections obtained as the difference between calculated and observed travel times for an M 5.3 aftershock of the Notohanto earthquake (colored squares). The crosses represent the first $24 \mathrm{~h}$ of aftershocks with magnitude larger than 2. A star depicts the JMA epicenter of the mainshock and a red rectangle the fault plane model used for imaging. Thin black lines represent faults in the region.

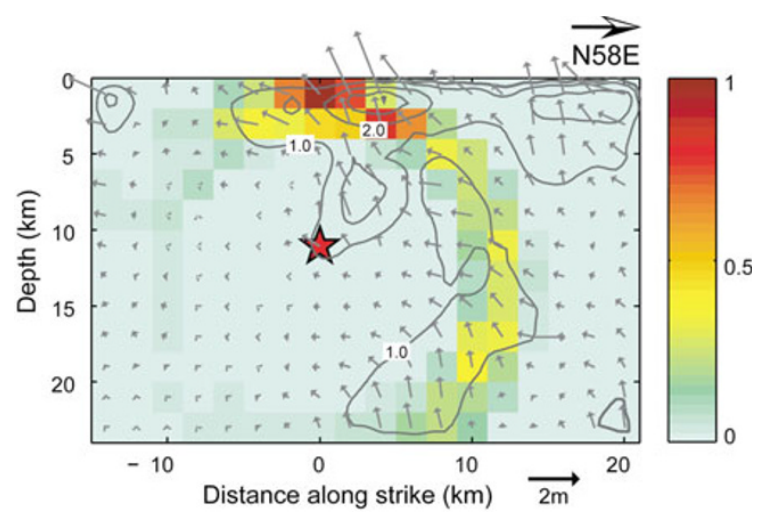

Fig. 5. Normalized fault plane brightness distribution obtained by the Isochrones Back-Projection Method (in a color scale). A slip model of the Notohanto earthquake obtained by an inversion of near-source strong ground motions is overlapped as black contour lines (every meter), and gray arrows, with a maximum slip of $4.3 \mathrm{~m}$ (Aoi and Sekiguchi, 2007). A red star depicts the rupture starting point. The fault configuration and grid spacing $(2 \mathrm{~km})$ is the same for both models.

are expected, and smaller towards stations within the Hida range (Fig. 4).

\subsection{Source image of the Notohanto earthquake}

We applied the procedures outlined in the previous sections to obtain an image of the total brightness distribution across the fault plane for the Notohanto earthquake (Fig. 5). In this case we have iterated the restarting procedure 25 times, which was obtained as an appropriate value for source imaging in our resolution test. Our results show two large brightness regions; the first region is located $10 \mathrm{~km}$ above the hypocenter along the dip, and the second region is observed at the bottom of the northern end of the fault plane (Fig. 5). These large brightness regions approximately correspond to large slip areas obtained in the source model by Aoi and Sekiguchi (2007) (Fig. 5), based on an in- version of near-source strong motion data (Sekiguchi et al., 2002). For the IBM source imaging we used the EW components, but the use of the NS component lead to a very similar source image result. We also tested the IBM source imaging of the Notohanto earthquake using the waveforms filtered in a low-frequency band $(0.1-1 \mathrm{~Hz})$, and in a broad frequency band $(0.1-30 \mathrm{~Hz})$, and found no significant difference to the result for the imaging using high-frequency waveforms. This result supports the idea that the IBM imaging results may approximately indicate the location of asperities of earthquakes. We subsequently will refer to large brightness regions as asperities. In Fig. 6 we show all $P$ wave velocity envelopes (EW component) used for source imaging, aligned at the $P$-wave onset and ordered by increasing arrivals times. We highlighted by gray vertical lines the arrival times corresponding to the largest asperity, by including the 1-s window used for the envelope averaging around the isochrones arrivals. We can observe in general that this window includes large envelope wavelets radiated from the asperity (Fig. 6). In order to explore the relationship between aftershocks and asperities, we overlapped the horizontal projection of source brightness image, and the first $24 \mathrm{~h}$ of aftershocks with magnitude larger than 2, obtained by a manual relocation of Hi-net events (Fig. 7). The fault rupture progressed bi-laterally and did not extend beyond $12 \mathrm{~km}$ towards the NE, and $6 \mathrm{~km}$ towards the SW along the strike. This fault rupture area corresponds approximately to a region with the largest concentration of aftershocks (Fig. 7).

\subsection{Rupture velocity and fault plane geometry}

Using the methodology examined in the resolution test we calculated the total fault plane brightness for different rupture velocity values, as shown in Fig. 8. We can observe that the fault plane brightness has a clear peak for a rupture velocity value of $2.3 \mathrm{~km} / \mathrm{s}$, which would correspond to the optimum value for the Notohanto earthquake. To explore the stability of the solution, we tested different fault plane models by changing the fault strike to $63^{\circ}$ and the fault dip to $60^{\circ}$, with respect to the F-NET solution. In all cases the optimum rupture velocity value is close to $2.3 \mathrm{~km} / \mathrm{s}$ (Fig. 8). In the case of the asperity distribution result we found that modifying the strike by $5^{\circ}$ (with respect to the FNET solution) did not significantly affect the source image solution, whereas a change by $6^{\circ}$ in dip (with respect to the F-NET solution) introduced a defocusing effect into the source image (Fig. 9). This would mean that the fault plane corresponding to the F-NET solution leads to a better, more focused source image compared with the solutions for other fault plane geometries. For all the models tested we found in general that the rupture velocity value estimated by IBM is very stable.

\subsection{Source image computation time}

One of the main advantages of the IBM source imaging is its very fast computation time. The calculation of a source model using IBM mainly involves computation of travel times and the source imaging itself. The CPU time required to calculate all the travel times for the Notohanto earthquake is about $10 \mathrm{~min}(1 \mathrm{CPU})$. In the case of source imaging, the calculation is done within $1 \mathrm{~min}$. On the other hand the calculation of a source model of the Notohanto earthquake 

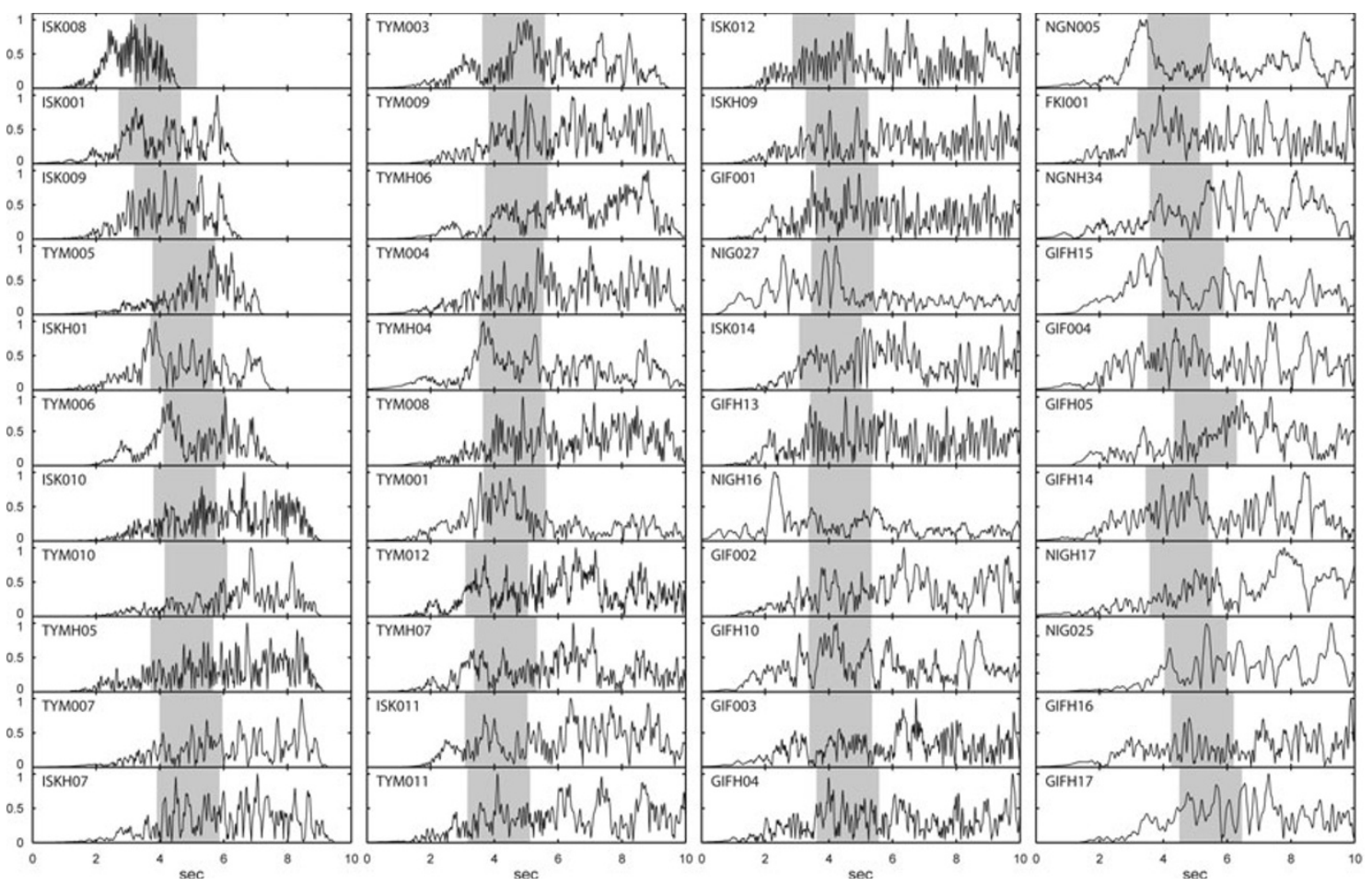

Fig. 6. EW component of velocity envelopes at K-NET and KiK-net sites used for source imaging, calculated from bandpass-filtered waveforms (1 to $30 \mathrm{~Hz}$ ). The envelopes are aligned at the $P$-wave onset and ordered by increasing arrivals. The thick gray line represent the isochrone time corresponding to the largest asperity, by including $\mathrm{a}+/-1 \mathrm{~s}$ window around the isochrone.

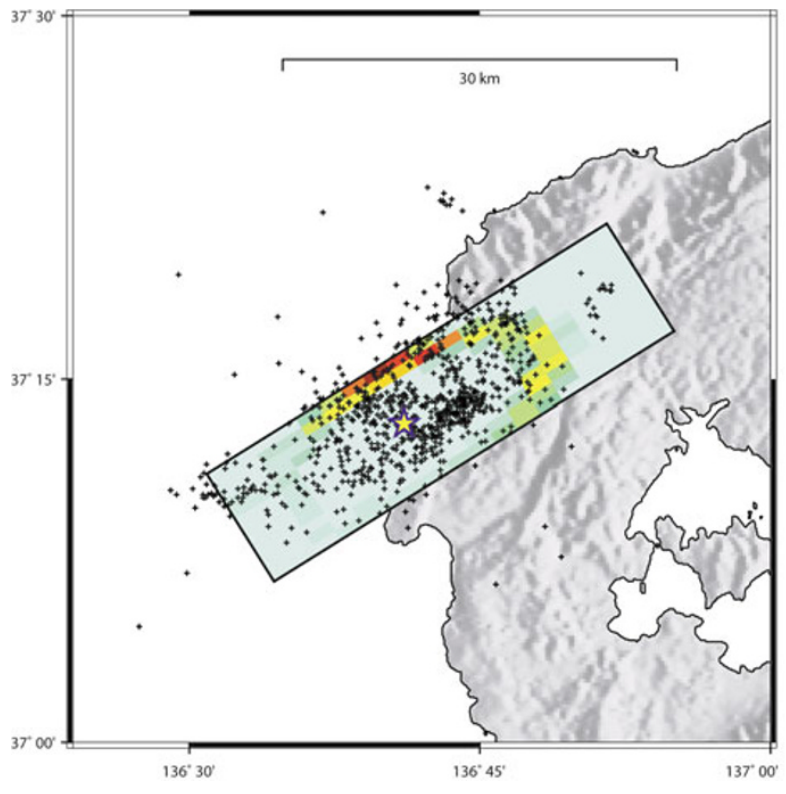

Fig. 7. Horizontal projection of the fault plane brightness (color scale), overlapped by the first $24 \mathrm{~h}$ of aftershocks with magnitude larger than 2 (black crosses). A yellow star depicts the JMA epicenter of the mainshock and a black rectangle the fault plane model used for source imaging.

using a conventional inversion procedure requires several hours of CPU time (Aoi and Sekiguchi, 2007). The total CPU time required for the IBM source imaging may be further reduced by calculating in advance tables of travel times for variable distances and source depths, at every KNET and KiK-net station. This would make IBM ideal for

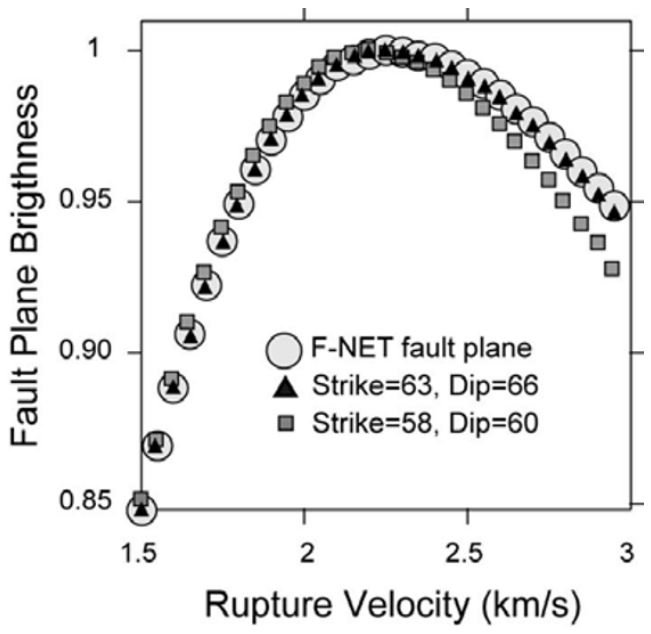

Fig. 8. Normalized total fault plane brightness vs fault rupture velocity. Source imaging solutions for three different fault plane geometries are compared: circles correspond to the F-NET fault plane solution $\left(M_{\mathrm{w}} 6.7\right.$, strike of $58^{\circ}$ and a dip of $\left.66^{\circ}\right)$, triangles correspond to a fault plane with a strike of $63^{\circ}$ and a dip of $66^{\circ}$, and squares to a strike of $58^{\circ}$ and a dip of $60^{\circ}$.

quasi-real time estimation of an initial source process of earthquakes.

\section{Conclusions}

-We investigated the source process of the 2007 Notohanto earthquake by applying a new source imaging approach, the Isochrones Back-Projection Method (IBM), which directly maps high-frequency seismogram envelopes into an image of the source rupture. 

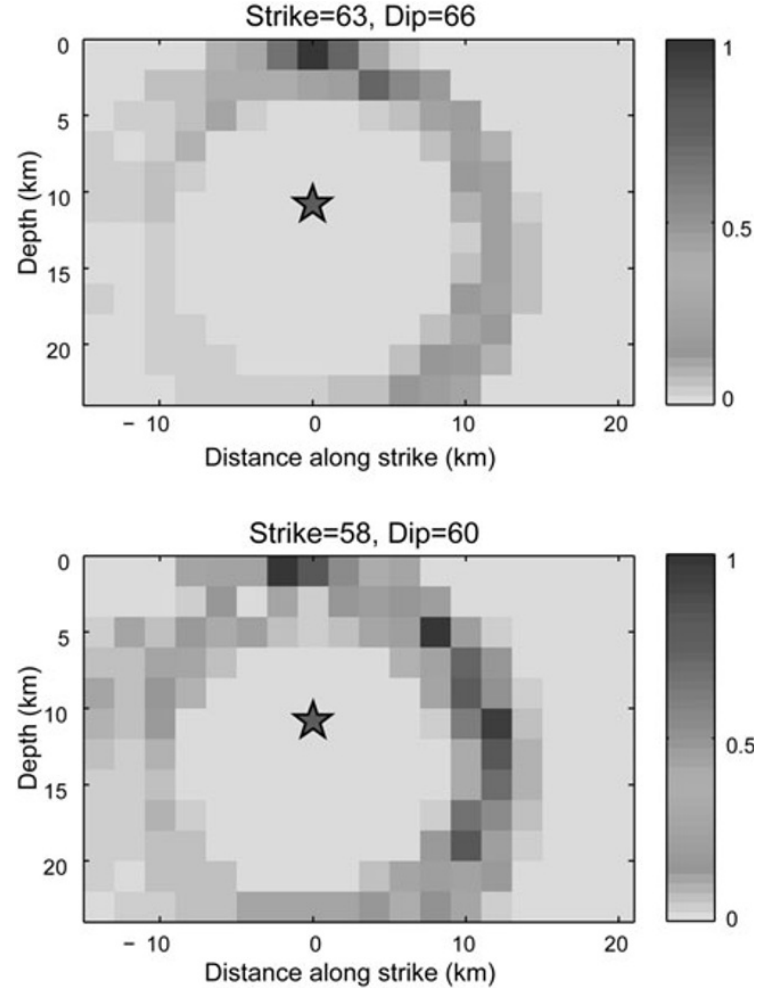

Fig. 9. Fault plane brightness solution for a fault plane with a strike of 63 and a dip of $66^{\circ}$ (upper), and a fault plane with a strike of $58^{\circ}$ and a dip of $60^{\circ}$ (lower)

-Our source image of the Notohanto earthquake shows two large brigthness regions; the first region is located $10 \mathrm{~km}$ above the hypocenter along the dip and the second region is located near the bottom of the fault, NE of the hypocenter. These large brightness regions approximately correspond to large slip areas obtained by a standard source inversion approach. Our results demonstrate that IBM has the capability to quickly map asperities of large earthquakes using observed strong motion data and, therefore, could be suitable for a near-real time estimation of the source process of earthquakes.

-The rupture area of the Notohanto earthquake extended bilaterally $12 \mathrm{~km}$ towards the NE, and $6 \mathrm{~km}$ towards the SW along the strike. This fault area approximately corresponds with the largest concentration of aftershocks.

-We obtained an optimum fault rupture velocity value for the Notohanto earthquake of $2.3 \mathrm{~km} / \mathrm{s}$. Our results show that the IBM is able to provide stable estimates of fault rupture velocity.

-A sensibility analysis on the influence of fault geometry on the source imaging result shows that the fault plane corresponding to the F-NET solution provides a well focused source image, compared with the results from other fault plane geometries.

-The results of a resolution test show that the IBM is able to identify the location of large slip areas of earthquakes.
However, further studies are required to improve the accuracy of the imaged sources, as well as the estimations of fault rupture velocity.

Acknowledgments. We would like to thank Prof. K. Ito for kindly providing the velocity model values used in this study, Dr. M. Matsubara for providing manual Hi-net locations of the Notohanto earthquake aftershocks, and associate editor Dr. T. Iidaka. This work was conducted under the NIED project "Strong Motion Prediction and Seismic Hazard Evaluation", and the Kakenhi Grant No. 18540428. We appreciate the comments of two anonymous referees. All the computations for this study were carried out in an HP Alpha Server GS1280 at NIED.

\section{References}

Allmann, B. and P. M. Shearer, A High-Frequency Secondary Event During the 2004 Parkfield Earthquake, Science, 318, 1279-1283, 2007.

Aoi, S. and H. Sekiguchi, Rupture process of the 2005/3/25 NotoHanto Earthquake by using Near-fault dat a (preliminary version), http://www.kyoshin.bosai.go.jp/k-net/topics/noto070325/, 2007.

Bernard, P. and R. Madariaga, A New Asymptotic Method for the Modeling of Near-Field Accelerograms, Bull. Seismol. Soc. Am., 74, 539-557, 1984.

Ellsworth, W. L., Imaging Fault Rupture Without Inversion, Seismol. Res Lett., 63, 73, 1992.

Festa, G., Slip imaging by isochron back projection and source dynamics with spectral element methods, Doctor thesis, University of Bologna and National Institute of Geophysics and Volcanology, pp. 195, 2004.

Festa, G. and A. Zollo. Fault slip and rupture velocity inversion by isochrone backprojection, Geophys. J. Int., 166, 745-756, 2006.

Fletcher, J. B., P. Spudich, and L. M. Baker, Rupture Propagation of the 2004 Parkfield, California, Earthquake from Observations at the UPSAR, Bull. Seismol. Soc. Am., 96, S129-S142, 2006.

Hamada, M., O. Aydan, and A. Sakamoto, A quick report on Noto Peninsula earthquake on March 25, 2007, http://www.jsceint.org/Report/Noto_earthquake/Noto_report.pdf, Japan Society of Civil Engineers, 2007.

Hartzell, S. and T. H. Heaton, Inversion of Strong Ground Motion and Teleseimic Waveform Data for the Fault Rupture History of the 1979 Imperial Valley, California Earthquake, Bull. Seismol. Soc. Am., 73, 1553-1583, 1983.

Ishii, M., P. Shearer, H. Houston, and J. E. Vidale, Extent, duration and speed of the 2004 Sumatra-Andaman earthquake imaged by the Hi-Net array, Nature, 435, 933-936, 2005.

Ito, K. and H. Wada, Observation of microearthquakes in the Atotsugawa fault region, central Honshu, Japan seismicity in the creeping section of the fault, in Seismogenic Process Monitoring, edited by H. Ogasawara, T. Yanagidani, and M. Ando, 229-243, A. A. Balkema Publishers, Rotterdam, 2002

Iwata, T. and K. Irikura, Tomographic Imaging of Heterogeneous Rupture Process of a Fault Plane, Jisin, 42, 49-58, 1989.

Kao, H. and S. J. Shan, Rapid identification of earthquake rupture plane using Source-Scanning Algorithm, Geophys. J. Int., 168, 1011-1020, 2007.

Sekiguchi, H., K. Irikura, and T. Iwata, Source inversion for estimating the continuous slip distribution on a fault-introduction of Green's functions convolved with a correction function to give moving dislocation effects in subfaults, Geophys. J. Int., 150, 377-391, 2002.

Spudich, P. and L. N. Frazer, Use of Ray Theory to Calculate Highfrequency Radiation from Earthquake Sources having Spatially Variable Rupture Velocity and Stress Drop, Bull. Seismol. Soc. Am., 74, 20612082, 1984.

Spudich, P. and E. Cranswick, Direct observation of rupture propagation during the Imperial Valley earthquake using a short baseline accelerometer array, Bull. Seismol. Soc. Am., 74, 2083-2114, 1984.

N. Pulido (e-mail: nelson@bosai.go.jp), S. Aoi, and H. Fujiwara 